Document downloaded from:

http://hdl.handle.net/10251/82087

This paper must be cited as:

Fuentes Muela, M.; Vargas, D.; Gómez Barquero, D. (2016). Low-Complexity Demapping Algorithm for Two-Dimensional Non-Uniform Constellations. IEEE Transactions on Broadcasting. 62(2):375-383. doi:10.1109/TBC.2015.2492477.

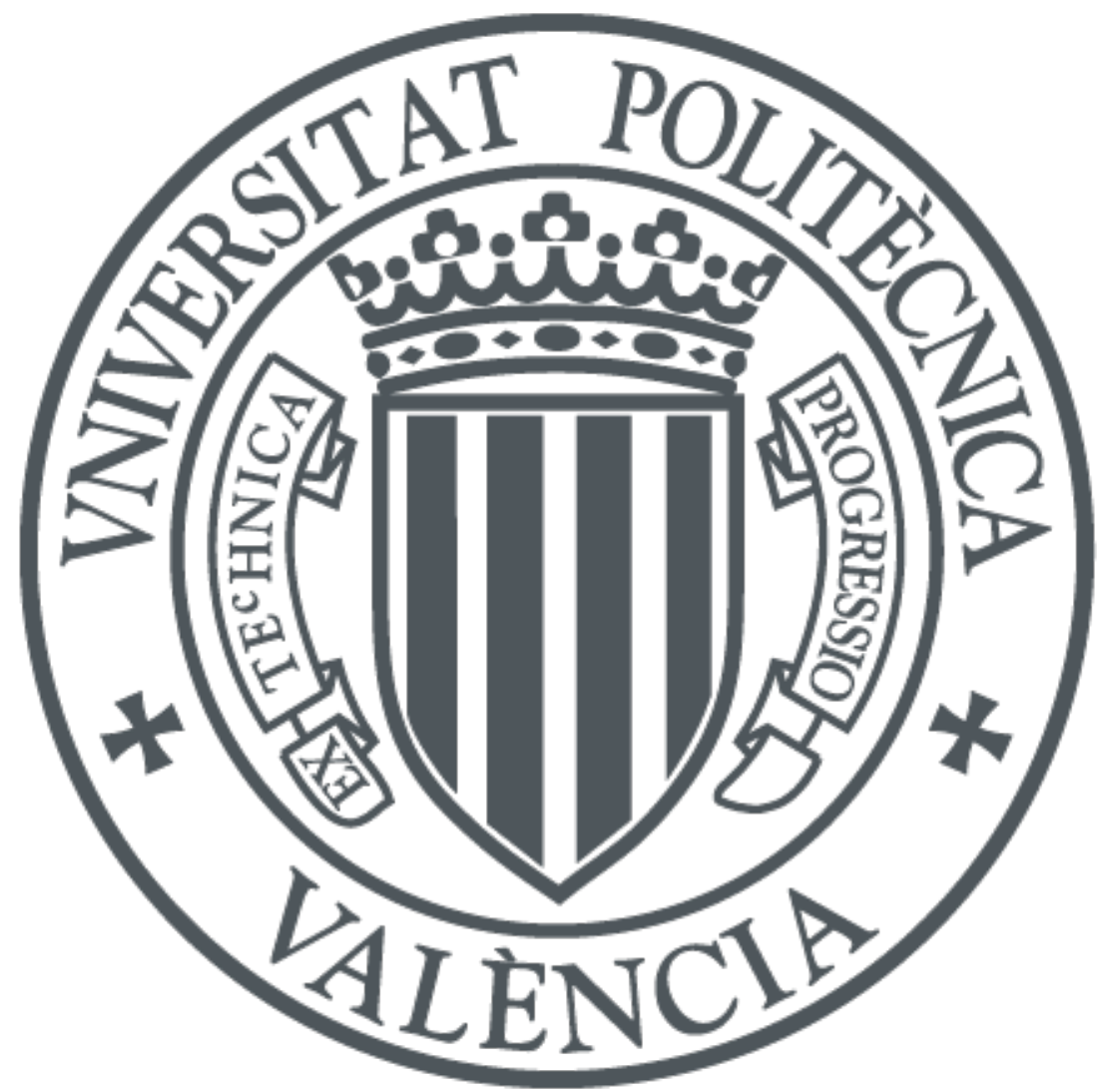

The final publication is available at

http://dx.doi.org/10.1109/TBC.2015.2492477

Copyright Institute of Electrical and Electronics Engineers (IEEE)

Additional Information

"(c) 2015 IEEE. Personal use of this material is permitted. Permission from IEEE must be obtained for all other users, including reprinting/ republishing this material for advertising or promotional purposes, creating new collective works for resale or redistribution to servers or lists, or reuse of any copyrighted components of this work in other works.") 


\title{
Low-Complexity Demapping Algorithm for Two-Dimensional Non-Uniform Constellations
}

\author{
Manuel Fuentes, David Vargas, and David Gómez-Barquero
}

\begin{abstract}
Non-uniform constellations (NUC) have been recently introduced in digital broadcasting systems to close the remaining gap to the unconstrained Shannon theoretical limit. Compared to uniform quadrature amplitude modulation (QAM) constellations, NUCs provide a signal-to-noise ratio (SNR) gain (i.e., a reduction in the required SNR), especially for high-order constellations. One-dimensional NUCs (1D-NUC) have a squared shape with non-uniform distance between the constellation symbols. Since the $I$ and $Q$ components remain as two independent signals, a 1D-demapper as for uniform QAM constellations is feasible. Two-dimensional NUCs (2D-NUC) provide a better performance than 1D-NUCs, since they are designed by relaxing the square shape constraint, with arbitrary shape along the complex plane. However, the main drawback of 2D-NUCs is the higher complexity at the receiver, since a 2D-demapper is needed. In this paper, we propose a demapping algorithm that reduces from $69 \%$ to $93 \%$ the number of required distances when using 2D-NUCs. The algorithm discards or replicates those constellation symbols that provide scarce information, with a performance degradation lower to $0.1 \mathrm{~dB}$ compared to the optimal Maximum Likelihood (ML) demapper.
\end{abstract}

Index Terms-Broadcasting, Non-Uniform Constellations, Demapping Complexity, ATSC 3.0.

\section{INTRODUCTION}

B IT-INTERLEAVED Coding and Modulation (BICM) is the pragmatic approach for combining channel coding and digital modulations in fading transmission channels [1]. The core of the BICM encoder consists of the serial concatenation of a forward error correcting (FEC) code, a bit interleaver (BIL), and a mapper that allocates blocks of bits to the constellation symbols. Nowadays, state-of-the-art BICM broadcasting systems are currently very close to the unconstrained Shannon theoretical limit [2].

Regarding the evaluation of the modulations, first generation terrestrial broadcasting systems such as DVB-T (Digital Video Broadcasting - Terrestrial) [3] or ISDB-T (Integrated Services Digital Broadcasting) [4] support QPSK, 16QAM and 64QAM uniform constellations. The current state-of-the-art terrestrial broadcasting technology commercially deployed DVBT2 (Terrestrial 2nd Generation) [5] includes the 256QAM modulation and supports rotated constellations (RC). With $\mathrm{RCs}$, a certain rotation angle is applied to the constellation,

Manuscript received July 6, 2015; reviewed September 24, 2015; accepted September 28, 2015. This work is defined in the "Programa de Valoración y Recursos Conjuntos de I+D+i de VLC/CAMPUS" and founded by the Ministerio de Educación, Cultura y Deporte as part of the "Programa Campus de Excelencia Internacional".

M. Fuentes, D. Vargas and D. Gómez-Barquero are with the Institute of Telecommunications and Multimedia Applications (iTEAM) of the Universitat Politecnica de Valencia, 46022 Valencia, Spain (e-mail: \{mafuemue, davarpa, dagobar\}@iteam.upv.es). so that the binary information is transmitted simultaneously in different in-phase (I) and quadrature (Q) components. In order to ensure that each component undergoes independent fading, a component interleaver is needed after the rotation [6]. The handheld evolution of DVB-T2, NGH (Next Generation Handled), is the first broadcasting system which includes onedimensional non-uniform constellations (1D-NUC), for 64 and 256NUC orders [7]. The next-generation U.S. terrestrial broadcasting standard ATSC 3.0 (Advanced Television Systems Committee 3rd Generation) includes two-dimensional nonuniform constellations (2D-NUC) from 16 to 256NUC, and 1D-NUCs for new high-orders such as 1024NUC (or 1kNUC) and 4096NUC (or 4kNUC) [2].

With uniform QAM, the constellation symbols are regularly spaced in the constellation diagram and transmitted with equal probabilities. However, there is a gap between the BICM capacity of uniform QAM constellations and the theoretical Shannon limit, which increases with the modulation order. NUCs can be used to reduce this gap and provide a better performance, reducing the required SNR with respect to the corresponding uniform constellation (i.e., providing a coverage gain). Consequently, the potential gain provided by NUCs increases with the constellation size. NUCs are designed for a particular SNR, and for a specific channel. For strong error correcting codes such as Low-Density Parity Codes (LDPC), with steep bit error rate (BER) curves as a function of the SNR, the target SNR of the NUC is easily selected for each code according to the SNR of the waterfall region.

1D-NUCs have a squared shape with non-uniform distance between the constellation symbols, and they are formed by two non-uniform pulse amplitude modulation (PAM) signals [8]. Hence, the complexity can be drastically reduced by using a one-dimensional demapper [9]. It utilizes the mapping feature that the original constellation symbols in the same I/Q component belong to the same subset for a specific even or odd bit ( 0 or 1$)$. The idea is to only select the best candidate in each component and to find the minimum Euclidean distance.

2D-NUCs are designed by relaxing the square shape constraint of uniform constellations, with a better performance than 1D-NUCs but with a higher receiver complexity since they cannot be separated into two independent I/Q components. In addition, the constellations adopted by ATSC 3.0 retain left-right and up-down symmetry [10]. Hence, the complete constellation can be derived by defining just the first quarter of the complex symbols. Fig. 1 shows an example of 2D-256NUCs designed for three different SNRs of 10, 18 and $26 \mathrm{~dB}$, for i.i.d. (independent and identically distributed) Rayleigh channel. When optimizing NUCs at high SNRs the 

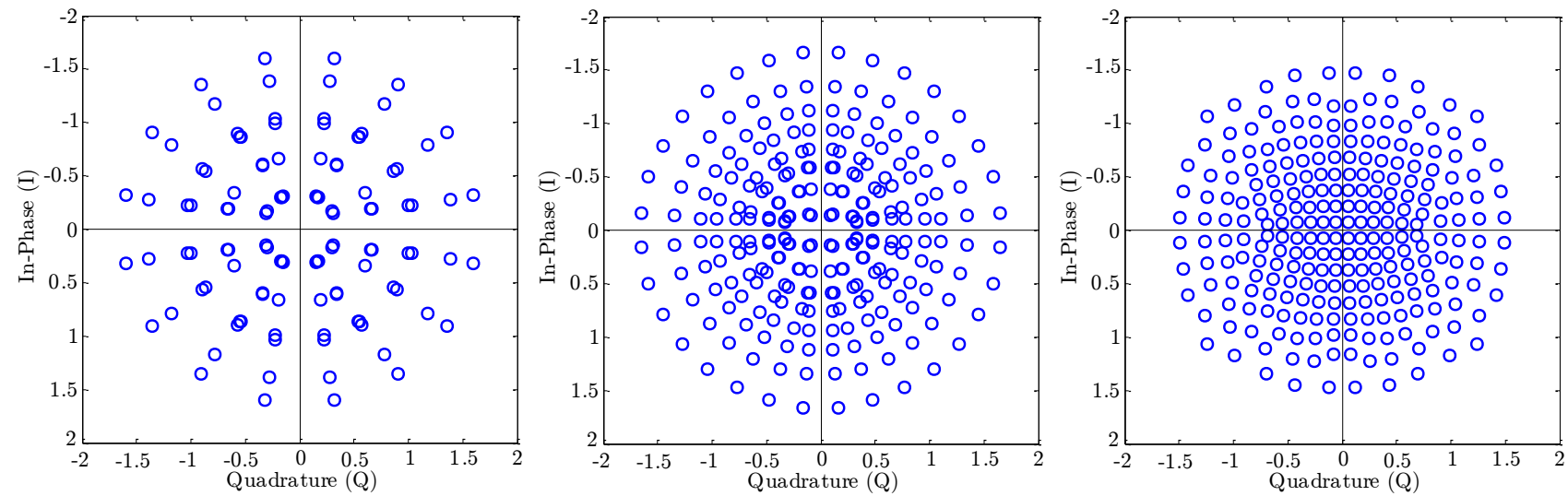

Fig. 1. Result of 2D-256NUCs designed for CRs 5/15 (left), 9/15 (center) and 13/15 (right), optimized for SNRs of 10, 18 and $26 \mathrm{~dB}$, respectively, i.i.d. Rayleigh channel.

positions converge toward the uniform QAM values. Without a robust FEC code, the best option is to pack the constellation symbols as uniformly spaced as possible. At low SNRs, NUCs collapse into lower orders of constellation [11], where almost identical symbols are grouped in clusters. With the condensation, the most significant bits (MSB) provide similar robustness as the positions of low-order constellations. On the other hand, the least significant bits (LSB) cannot be resolved from the overlapping points, since they offer a very weak information, close to zero [12].

Previous works in the literature outline the design and the evaluation of potential gains of NUCs. Reference [13] noted the capacity shortfall for uniform QAMs, and introduced the non-uniform concept, obtaining several constellations which offer a capacity improvement. A more recent study [11] tackles the optimization of 1D-NUCs, in AWGN channel. In [14], high-order 1D- and 2D-NUCs are optimized with respect to their BICM capacity, also for AWGN channel. In [15], high-order NUCs with constellation sizes of up to 4kQAM are investigated for Ultra-High Definition TV (UHDTV) broadcasting services.

One of the bottlenecks in real receiver implementations is the use of demappers that calculate the distances to all constellation symbols. This paper proposes a generic demapping algorithm that can be applied to any type of 2D-NUCs. As an example of application, we provide the results for the NUC constellations optimized for ATSC 3.0. The paper focuses on the $256 \mathrm{NUC}$ constellation, which is the highest modulation order of 2D-NUCs used in the ATSC 3.0 specification [10]. As an additional result, the approach is also extended to a higher constellation order of 2D-1kNUC. Code rates from $2 / 15$ to $13 / 15$ are considered. The proposed algorithm is based on two different strategies. The first one takes advantage of the symmetry that constellations provide, selecting a cluster of points to compute the LLRs. It is based on the demappers proposed in [16] and [17] for RCs. The concept is similar to the Sphere Demapper (SD) [18], which also selects a cluster of points to compute the LLRs. From the received point, SD only selects the constellation points that are inside a fixed radius. With SD, the number of operations is significantly reduced compared to the Maximum Likelihood (ML) demapper. On the other hand, reference [19] also analyzes the quadrant symmetry of the constellations, in order to precompute the LLR values in a look-up table and completely avoid the demapping process. The second strategy exploits the condensation of NUCs, especially at low SNRs where some of the constellation symbols almost repeat the same position in the I-Q plane. A demapper exploiting this condensation was first proposed in [20] and analyzed in [21]. Both strategies can be combined in order to reduce from $69 \%$ to $93 \%$ the number of required distances, depending on the $\mathrm{CR}$, with almost no performance loss compared to the optimal ML demapper. The proposed demapper can be combined with RCs, in order to improve the SNR requirement for high CRs with no additional complexity.

The rest of the paper is structured as follows. Section II describes the demapping process of 2D-NUCs and its complexity implications. Section III proposes an algorithm designed to reduce the demapping complexity. Section IV presents results in terms of performance, for a complete range of CRs. Finally, the main findings of the work are summarized in Section V.

\section{Demapping Complexity at the Receiver}

This analysis is limited to single-input single-output (SISO) antenna systems. It is necessary to differentiate between two elements when comparing complexity: the number of required distances to calculate each LLR and the complexity for the calculation of the distance itself. Hence, the metric is calculated with a different dimensionality for $1 \mathrm{D}$ and $2 \mathrm{D}$ demapping. From the received symbol vector $y$, and the channel vector $h$, each log-likelihood ratio (LLR) $\Lambda_{l}$ is computed for all code bits $c_{l}, l=1, \ldots, B$, with $B$ as the number of bits that affect each dimension of a constellation. $B$ does not refer to the number of bits per cell $(b p c)$. For the LLR computation, a total number of $N$ Euclidean distances between the received symbol $y$ and all constellation symbols $x$ is calculated. A single output $\Lambda_{l}$ can be computed with the following expression:

$$
\Lambda_{l} \triangleq \log \frac{p\left(c_{l}=1 \mid y, h\right)}{p\left(c_{l}=0 \mid y, h\right)}=\log \frac{\sum_{x \in \chi_{l}^{1}} \exp \left(-\frac{|y-h x|^{2}}{\sigma_{v}^{2}}\right)}{\sum_{x \in \chi_{l}^{0}} \exp \left(-\frac{|y-h x|^{2}}{\sigma_{v}^{2}}\right)}
$$


TABLE I

DEMAPPING COMPLEXITY OF UNIFORM QAM, 1D-NUC, 2D-NUC AND RC, FOR DIFFERENT BITS PER CELL, IN TERMS OF MATHEMATICAL OPERATIONS TO COMPUTE THE LLRS.

\begin{tabular}{|l||c|c|c|c|c|}
\hline bpc & 4 & 6 & 8 & 10 & 12 \\
\hline \hline QAM & 4 & 8 & 16 & 32 & 64 \\
\hline 1D-NUC & 4 & 8 & 16 & 32 & 64 \\
\hline 2D-NUC & 32 & 128 & 512 & 2048 & 8192 \\
\hline RC & 32 & 128 & 512 & 2048 & 8192 \\
\hline
\end{tabular}

where $\sigma_{v}^{2}$ represents the noise variance, $y$ is the received symbol, $x$ is a possible transmitted symbol, $h$ is the channel fading coefficient, and log refers to the natural logarithm. $\chi_{l}^{1}$ and $\chi_{l}^{0}$ denote the complementary sets of transmit vectors for which $c_{l}=1$ and $c_{l}=0$ respectively. The amount of complementary sets $\chi_{l}^{1}$ and $\chi_{l}^{0}$ has to be $B$. A Maximum Likelihood (ML) optimal demapper has therefore to consider $2^{B}$ symbols in a $D$-dimensional (real-valued) space. Hence, the complexity is $\mathcal{O}\left(D \cdot 2^{B}\right)$.

The complexity depends on the type of constellation. Uniform QAM constellations can be split into two PAM constellations. Therefore, the demapper has to consider the half of symbols $\left(B=\frac{b p c}{2}\right)$ in one dimension $(D=1)$. In this case, the complexity is $\mathcal{O}\left(2^{\frac{b p c}{2}}\right)$. With $1 \mathrm{D}-\mathrm{NUCs}$, this one-dimensional demapping is also possible, and the complexity is maintained, i.e., $\mathcal{O}\left(2^{\frac{b p c}{2}}\right)$. However, with $2 \mathrm{D}-\mathrm{NUCs}$ the demapper has to evaluate for all symbols $(B=b p c)$, the distances in two dimensions (real and imaginary parts, $D=2$ ). The complexity in this case is $\mathcal{O}\left(2^{b p c+1}\right)$.

The same occurs with RCs, where the binary information is transmitted simultaneously in different I and Q components. The demapper has to consider all symbols in two dimensions, regardless of the constellations shape. With RCs, it is not possible to use a 1D-demapper in any case. Therefore, the complexity with rotated constellations is also $\mathcal{O}\left(2^{b p c+1}\right)$. Table I shows the demapping complexity in terms of mathematical operations to compute the LLRs, depending on the type of constellation and bits per cell used.

The complexity order with 2D-NUCs becomes especially high from $b p c=8$. The proposed demapping algorithm reduces the number of distances $N$ necessary to compute each LLR with this type of constellations, and it is explained next.

\section{Proposed Algorithm to Reduce the Demapping COMPLEXITY OF 2D-NUCS}

The algorithm is based on two different strategies. The first strategy is called Quadrant Search Reduction (QSR), and computes the LLRs discarding those distances whose probability of being computed is significantly low. The second strategy is called Condensed Symbols Reduction (CSR), and omits the computation of those distances which are similar to others already calculated, and replicates them.

\section{A. Quadrant Search Reduction (QSR)}

The first strategy discards those distances that provide scarce information to the LLR computation. In this paper we apply QSR for 2D-NUCs. We note that the QSR algorithm can be
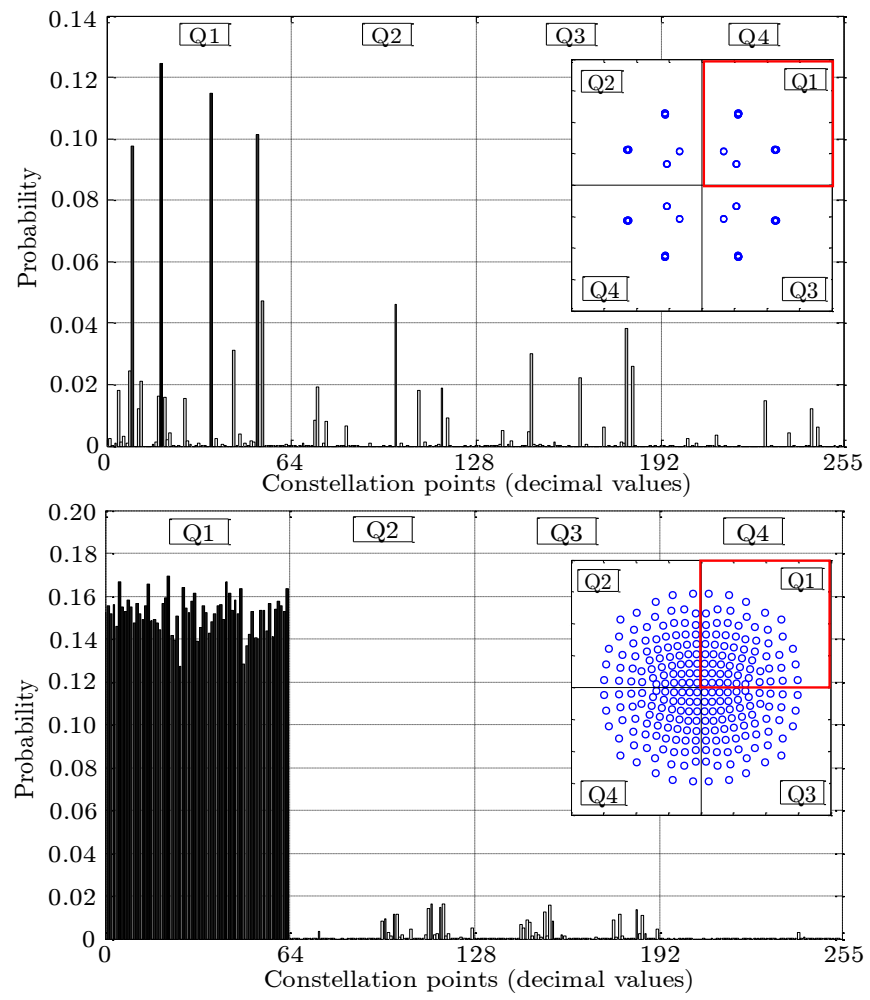

Fig. 2. Histogram of the received constellation symbols for $2 \mathrm{D}-256 \mathrm{NUC}$ in i.i.d. Rayleigh channel, with code rates 2/15 (top) and 13/15 (bottom).

used with any quadrant-symmetric constellation. The algorithm is divided into two main steps. The first step consists of calculating the probability of each symbol received, when they are transmitted from a particular quadrant of the constellation. The probability (from 0 to 1 ) of receiving a particular constellation symbol is calculated as the number of times the symbol is received divided by the total number of transmitted symbols. Then, the received symbols are organized by probability order. A total number of $10^{9}$ points is transmitted, over an i.i.d. Rayleigh channel. This number is confirmed in [17], and provides enough accuracy in the calculations. The rest of quadrants can be derived by symmetry. This step can be done off-line, storing the symbols by probability order in a look-up table. Then, the algorithm can compute the distances to the most probable symbols $N$ when necessary.

Fig. 2 shows two examples of the histograms achieved for 2D-256NUC in the first stage, with CRs $2 / 15$ and $13 / 15$, designed in i.i.d. Rayleigh channel for SNRs of 3 and $26 \mathrm{~dB}$ respectively. Both histograms have been obtained for the first quadrant $(\mathrm{Q} 1)$. For a given CR, the transmission is done for the threshold SNR that provides a bit error rate (BER) of $10^{-4}$. As Fig. 2 shows, the probability of receiving a constellation point from the Q1 is higher for high SNRs (bottom). On the other hand, at low SNRs (top) and because of the high noise level, constellation points from other quadrants (especially Q2 and Q3) are received with a higher probability.

In the second step the minimum number of distances with significant probability, necessary to implement the algorithm, is obtained. In order to select the final subset $N$, it is necessary 


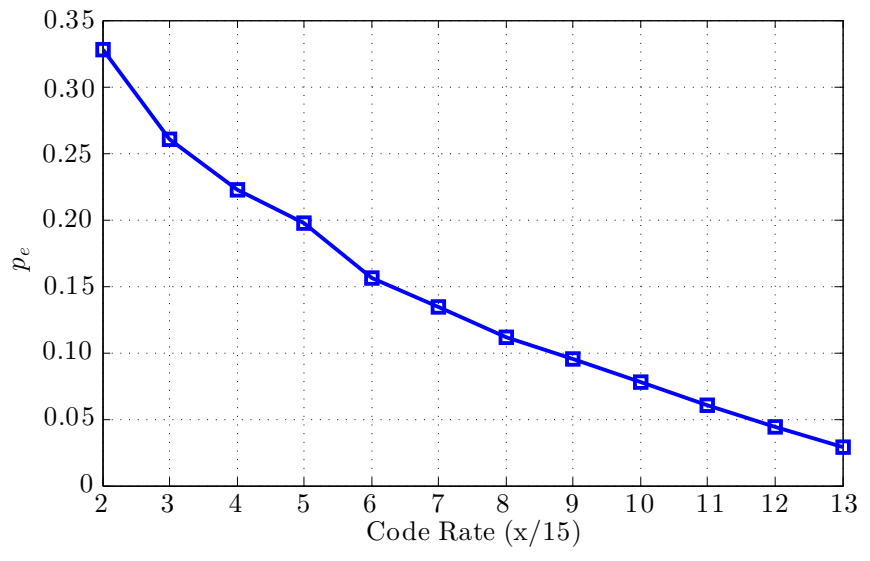

Fig. 3. Probability of selecting an erroneous quadrant $\left(p_{e}\right)$ with 2D-256NUCs in i.i.d. Rayleigh channel, with code rates from $2 / 15$ to $13 / 15$.

to observe its impact on the system performance through a BER analysis. The smaller the number of distances $N$ considered, the worse the performance, but with a lower complexity. The proposed criteria is to select the smallest possible $N$ that ensures a performance loss smaller than 0.1 $\mathrm{dB}$.

With QSR, it is assumed that a symbol is received in the same quadrant that was transmitted. Hence, it is necessary to know the transmitted quadrant. The quadrant is determined using the sign of the I/Q components of the received symbol. We define " $p_{e}$ " as the probability of selecting an erroneous quadrant. The constellation symbols that are in the edge of each quadrant provide less reliable results, and they usually lead to errors. The probability " $p_{e}$ " is higher for low CRs, with higher levels of noise power, see Fig. 3. However, precisely those constellations with a very high probability of obtaining an erroneous quadrant suffer a significant condensation. The second strategy, which is explained next, takes advantage of this condensation and calculates only distances for symbols that are not repeated.

\section{B. Condensed Symbols Reduction (CSR)}

When optimizing NUCs in a transmission system, it is possible to improve the BICM capacity by modifying the position of the QAM constellation symbols. At low CRs, NUCs converge to lower orders of constellation. In this case, the constellation symbols share the MSB, maximizing the Euclidean distance and maintaining independent dimensions for each bit, meanwhile the LSB have almost no impact in the LLR computation. The information provided by the LSB is close to zero and will remain so, even for very large SNRs [12]. These constellations are so-called condensed constellations, as the constellation symbols almost repeat the same position in the I-Q plane for the most nearby Gray mapped symbols [11]. For example, with a 2D-256NUC designed for a CR 2/15, which can be seen in the upper part of Fig. 2, only sixteen points are apparently visible, resembling a 16NUC. However, there are sixteen clusters with sixteen constellation points in almost identical positions.
CSR is based on this condensation and calculates a single distance for a complete cluster of symbols. The rest of distances are derived by replicating the previously calculated ones. In order to determine which symbols are grouped together and which not, it is necessary to define a minimum gap between them. The higher the gap selected, the bigger the number of symbols grouped together in a single distance to compute, reducing the complexity but also incurring into a higher performance degradation.

\section{Quadrant Condensed Search Reduction (QCSR)}

It is possible to combine both QSR and CSR. We call this algorithm Quadrant Condensed Search Reduction (QCSR). The algorithm consists of taking the $N$ constellation symbols obtained with QSR and group them together as done with CSR. The QCSR algorithm is detailed next.

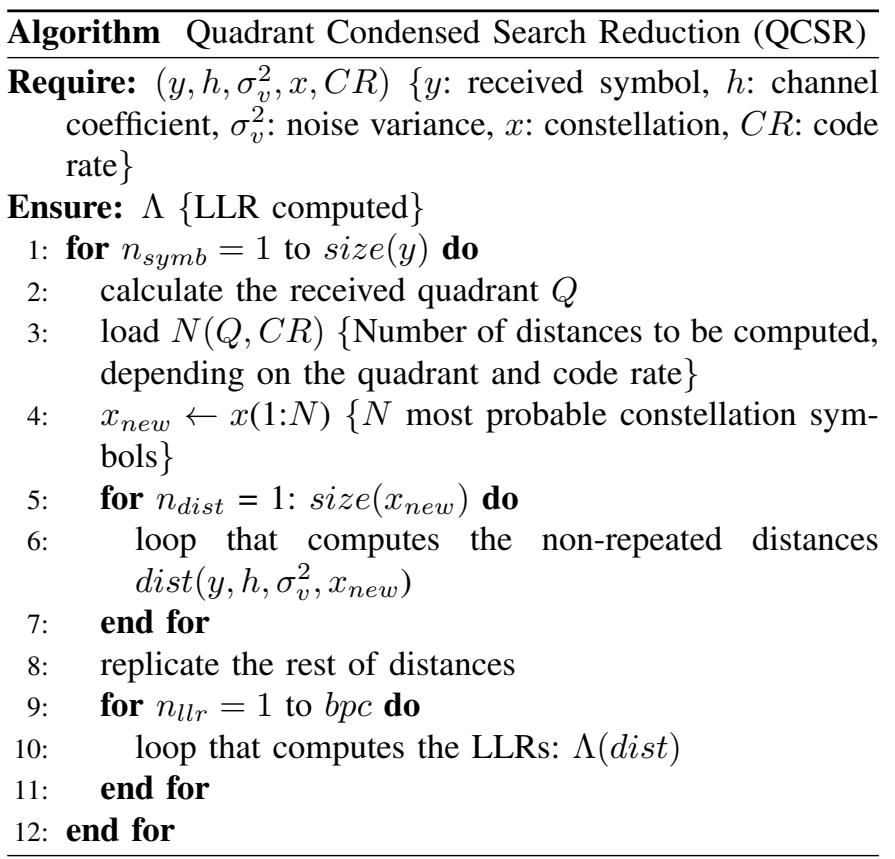

In the proposed algorithm, $y$ is the received symbol, $h$ is the channel fading coefficient, $\sigma_{v}^{2}$ represents the noise variance, $x$ is a possible transmitted symbol, and $C R$ refers to the code rate. $\Lambda$ denotes each log-likelihood ratio (LLR) computed.

At low SNRs, the CSR algorithm is more dominant because the optimized 2D-NUCs are condensed, which also complicates the quadrant search. At high SNRs the noise impact is lower and the constellations are not condensed, and hence the QSR algorithm is dominant.

\section{PERFormance EVAluation}

We compare first, in terms of BER performance, the results obtained with the optimal ML and the proposed QCSR demapper, and provide the minimum number of $N$ distances that need to be calculated, for i.i.d Rayleigh channel. Afterwards, the performance loss obtained for different channel models is provided. In the simulations, BILs and NUCs from ATSC 3.0 were used [2], with a LDPC code length of 64800 bits and FEC codes from $2 / 15$ to $13 / 15$. No time and frequency interleavers were applied in this case. 

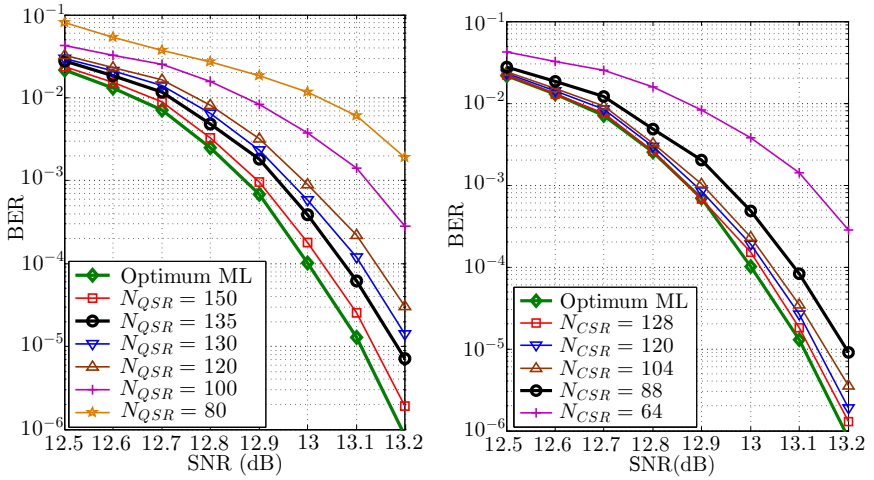

Fig. 4. Performance of QSR (left) and CSR (right) demappers, depending on the number of distances $N$ and compared with the ML demapper. Results presented for a code rate $6 / 15$, i.i.d. Rayleigh channel.

\section{A. Calculation of the minimum number of distances}

As an example, Fig. 4 shows the performance of the proposed strategies QSR and CSR for 2D-256NUCs and CR $6 / 15$, depending on the number of distances $N$. The selected criterion is to compare the SNR of each option that provides a BER of $10^{-4}$, selecting the smallest possible $N$ that ensures a performance loss smaller than $0.1 \mathrm{~dB}$. We define $N_{Q S R}, N_{C S R}$ and $N_{Q C S R}$ as the number of distances necessary for the QSR, CSR and QCSR demappers, respectively.

In this particular case, both strategies QSR and CSR provide a reasonable performance degradation compared to $\mathrm{ML}$, using a number of distances $N_{Q S R}=135$ and $N_{C S R}=88$. In this SNR range $(13 \mathrm{~dB})$, the noise impact is significantly low, allowing the QSR strategy to work. On the other hand, the constellation maintains a good condensation for CR 6/15, so the CSR strategy provides a high reduction. Moreover, both strategies can be combined. For the symbols obtained with QSR, only those distances that are not repeated are computed, and the rest are replicated, as CSR does. In this case, the reduction comes to $N_{Q C S R}=66$ distances.

Fig. 5 shows the performance comparison with the selected $N$ for four representative CRs: a low CR 2/15, a medium CR 6/15 (recently analyzed) and two high CRs 10/15 and 13/15. The idea is to show separately the contribution of each strategy to the proposed algorithm, observing the impact on the system performance.

With low CRs such as 2/15 the SNR required is very low, and then the constellations are compressed. The compression allows the CSR strategy to work especially well, obtaining a reduction in the number of distances from up to $N_{C S R}=16$, with no performance loss. However, precisely the low SNR hampers the quadrant search. The QSR strategy often is erroneous, and needs to calculate almost all distances, having $N_{Q S R}=235$. Therefore, in this particular case the QCSR algorithm works also with only $N_{Q C S R}=16$. Although the quadrant search fails, the algorithm only needs to consider the 16 unique distances that are not repeated, replicating the rest and providing the correct information to the LLR computation.

With the high CR $10 / 15$, the CSR strategy hardly works, as the constellations are barely condensed. With CSR, it is necessary to use $N_{C S R}=224$ distances. Nevertheless, the
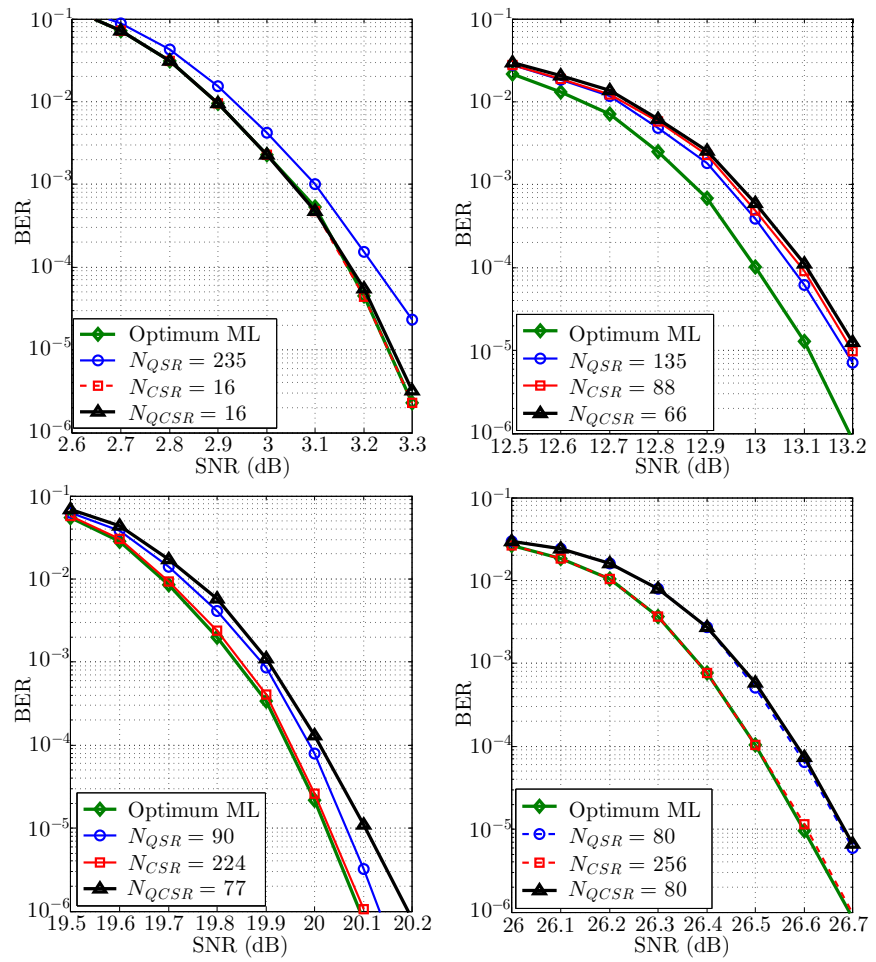

Fig. 5. Performance comparison of ML and QCSR demappers. QSR and CSR strategies are also shown by separate. Results presented for code rates 2/15 (top left), 6/15 (top right), 10/15 (bottom left) and 13/15 (bottom right), i.i.d. Rayleigh channel.

low noise impact allows QSR to work better, needing just $N_{Q S R}=90$. Combining both techniques, the final reduction is $N_{\text {QCSR }}=77$. On the other hand, the constellation optimized for the CR $13 / 15$ is not condensed at all, considering that each constellation symbol is unique in the I/Q plane. Hence, the QCSR algorithm is based only in the QSR strategy for this case, calculating $N_{Q C S R}=80$ distances.

Fig. 6 summarizes the minimum number of distances $N$ for the proposed algorithm, for all CRs from $2 / 15$ to 13/15. With CSR, the higher contribution is achieved for low CRs, where the number of operations can be reduced up to $N_{C S R}=16$, which represents a reduction of 93\%. Regarding the contribution of QSR, it can be seen that the curve is directly related with the probability of selecting an erroneous quadrant, $p_{e}$, shown in Fig. 3. However, the performance of QSR becomes better for high CRs, especially from $8 / 15$ to $13 / 15$. When combining the two strategies into one single algorithm, it becomes especially effective for medium CRs, where both advantages can be taken into account.

Fig. 7 shows the final reduced constellations used with QCSR when the first quadrant is transmitted, for the representative CRs analyzed: $2 / 15,6 / 15,10 / 15$ and $13 / 15$. In a real receiver, the complexity reduction is determined by the maximum number of distances $N$ to be computed. With the combined QCSR demapper, the maximum value was obtained for the CR 13/15, with $N_{Q C S R}=80$, which implies a reduction in the number of required operations of $69 \%$. 


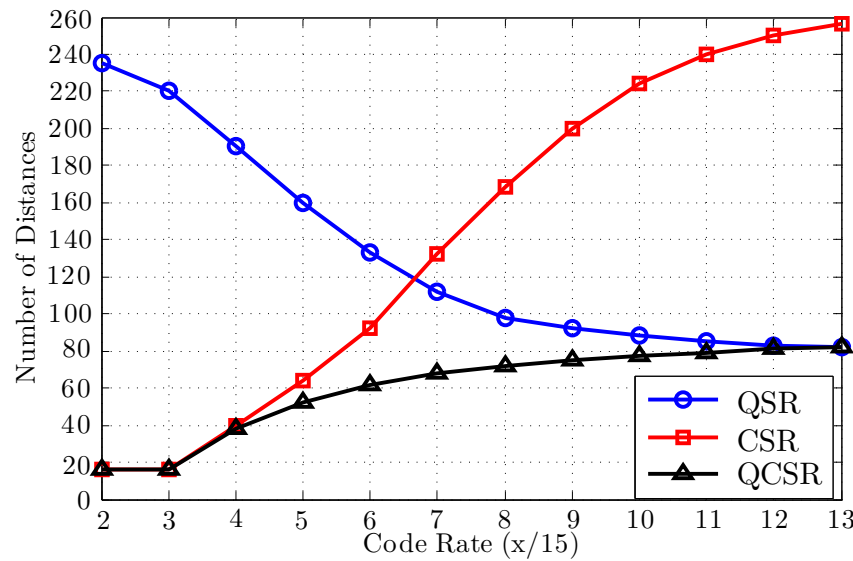

Fig. 6. Minimum number of distances $N$ required for both proposed algorithms, for $2 \mathrm{D}-256 \mathrm{NUC}$ and CRs from $2 / 15$ to $13 / 15$, i.i.d. Rayleigh channel.
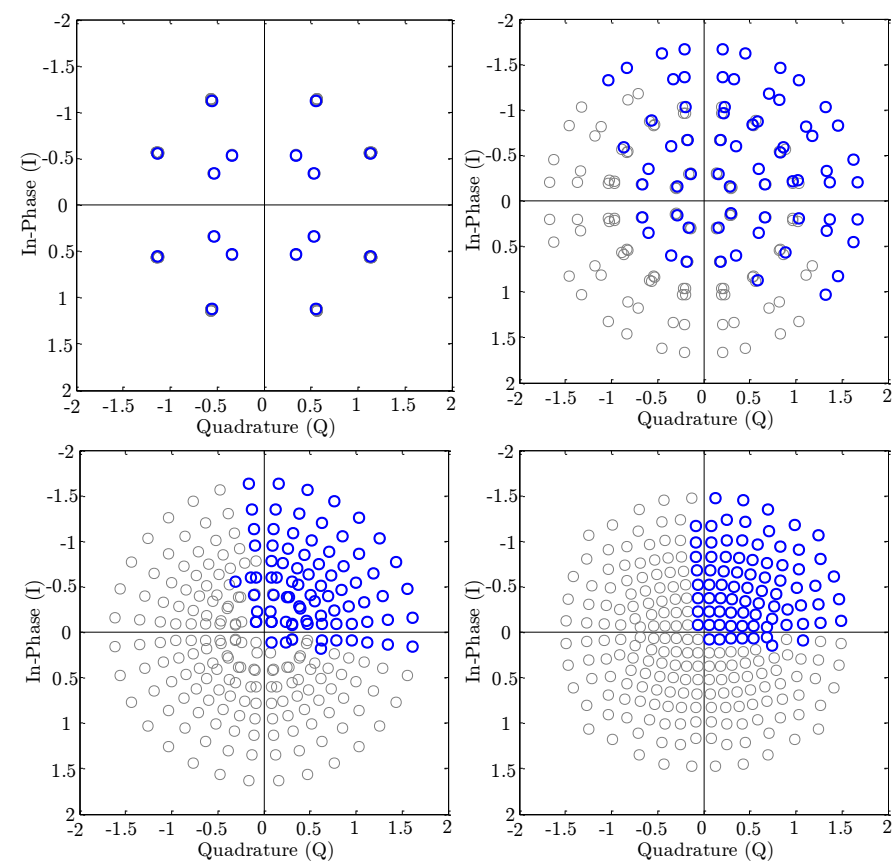

Fig. 7. Result of reduced 2D-256NUCs with QCSR, for CRs 2/15 (top left), 6/15 (top right), 10/15 (bottom left) and 13/15 (bottom right), i.i.d. Rayleigh channel.

\section{B. Performance Loss with Different Channel Models}

This section provides the performance loss of QCSR for different channel models for which the algorithm has not been optimized, under ideal and realistic channel estimation conditions. Table II shows the performance loss obtained with white Gaussian noise (AWGN), $F_{1}$ Ricean-fading and $P_{1}$ Rayleighfading channel models, which are defined in [22], compared to the results obtained in the analysis for i.i.d. Rayleigh channel model, under ideal channel estimation conditions. The $F_{1}$ channel is used to describe the fixed outdoor rooftop-antenna reception conditions, and the $P_{1}$ channel is used to describe the portable indoor or outdoor reception conditions.

With AWGN, the performance loss remains under $0.1 \mathrm{~dB}$ for all considered CRs. The SNR required is lower than
TABLE II

Performance Loss of QCSR Under Ideal Channel Estimation CONDITIONS

\begin{tabular}{|c|c|c|c|c|c|}
\cline { 3 - 6 } \multicolumn{2}{c|}{} & \multicolumn{5}{c|}{ Code Rate } \\
\hline Channel & Parameter (dB) & $2 / 15$ & $6 / 15$ & $10 / 15$ & $13 / 15$ \\
\hline \hline \multirow{2}{*}{ iid Rayleigh } & ML: SNR & 3.2 & 13 & 19.9 & 26.5 \\
\cline { 2 - 6 } & QCSR: Loss & $<0.1$ & $<0.1$ & $<0.1$ & $<0.1$ \\
\hline \multirow{2}{*}{ AWGN } & ML: SNR & 1.8 & 10.6 & 17.1 & 22.2 \\
\cline { 2 - 6 } & QCSR: Loss & $<0.1$ & $<0.1$ & $<0.1$ & $<0.1$ \\
\hline \multirow{2}{*}{$F_{1}$} & ML: SNR & 2.7 & 11.3 & 18 & 23.2 \\
\cline { 2 - 6 } & QCSR: Loss & $<0.1$ & $<0.1$ & $<0.1$ & $<0.1$ \\
\hline \multirow{2}{*}{$P_{1}$} & ML: SNR & 4.6 & 13.6 & 21.3 & 28.7 \\
\cline { 2 - 6 } & QCSR: Loss & $<0.1$ & 0.2 & 0.2 & 0.2 \\
\hline
\end{tabular}

TABLE III

PERFormance LosS OF QCSR UNDER REALISTIC CHANNEL ESTIMATION CONDITIONS

\begin{tabular}{|c|c||c|c|c|c|}
\cline { 3 - 6 } \multicolumn{2}{c|}{} & \multicolumn{4}{c|}{ Code Rate } \\
\hline Channel & Parameter (dB) & $2 / 15$ & $6 / 15$ & $10 / 15$ & $13 / 15$ \\
\hline \hline \multirow{2}{*}{$F_{1}$} & ML: SNR & 3.7 & 12.2 & 18.8 & 24 \\
\cline { 2 - 6 } & QCSR: Loss & $<0.1$ & $<0.1$ & $<0.1$ & $<0.1$ \\
\hline \multirow{2}{*}{$P_{1}$} & ML: SNR & 5.6 & 14.2 & 22 & 29.1 \\
\cline { 2 - 6 } & QCSR: Loss & $<0.1$ & 0.2 & 0.2 & 0.3 \\
\hline
\end{tabular}

the obtained for i.i.d. Rayleigh channel, allowing the QCSR algorithm to work better. The $F_{1}$ channel model with fixed reception, and therefore with a direct path (line-of-sight ray) also permits lower SNRs than the i.i.d. Rayleigh channel. For this reason, the performance loss of QCSR also remains under $0.1 \mathrm{~dB}$. However, the same does not occur with the $P_{1}$ portable channel. Having a less selective channel implies higher SNRs. Higher noise levels entail a worse result of the QCSR algorithm. In this case, the performance loss is up to $0.2 \mathrm{~dB}$ with high CRs.

Table III shows the performance loss of QCSR obtained under realistic channel estimation conditions. With real channel estimation, the channel fading $h$ received is different from the transmitted one. In order to obtain $h$, a linear frequency and time interpolation from the pilot pattern (PP) is done. In the case of study, PP7 and PP4 from DVB-T2 are used for the $F_{1}$ and $P_{1}$ channel models, respectively [22].

The higher performance loss of QCSR is obtained for the $P_{1}$ channel model, with $0.3 \mathrm{~dB}$ of difference in relation to the optimum ML demapper for the CR 13/15. These results confirm the robustness of the QCSR algorithm. QCSR requires, for all studied cases, SNRs nearby to the optimum ML demapper, feasible for its implementation in real receivers where the obtained performance loss is almost negligible.

\section{QCSR with Non-Uniform Rotated Constellations}

In this section, we show an example of application of the QCSR algorithm to non-uniform rotated constellations (NURC). First, we consider the (non-rotated) NUCs adopted in ATSC 3.0 and optimize the rotation angle, from QPSK to 256QAM. Selected angles are those that provide the maximum BICM capacity for the i.i.d. Rayleigh channel model. Afterwards, we analyze the SNR performance of the 2D-256NURCs with the proposed QCSR demapper, and compare it with the optimum ML demapper. 
TABLE IV

OPTIMUM ROTATION ANGLES $\left(^{\circ}\right)$ FOR 2D-NURCS WITH A MINIMUM ROTATION GAIN OF 0.1 DB, FOR I.I.D RAYLEIGH CHANNEL.

\begin{tabular}{|c||c|c|c|c|c|c|}
\hline Code Rate & $8 / 15$ & $9 / 15$ & $10 / 15$ & $11 / 15$ & $12 / 15$ & $13 / 15$ \\
\hline \hline QPSK & 23.6 & 25.5 & 26.4 & 27.1 & 27.4 & 27.6 \\
\hline 16NURC & - & - & 14.3 & 15.5 & 18.1 & 19.3 \\
\hline 64NURC & - & - & - & - & 2.5 & 12.1 \\
\hline 256NURC & - & - & - & - & - & 2.8 \\
\hline
\end{tabular}

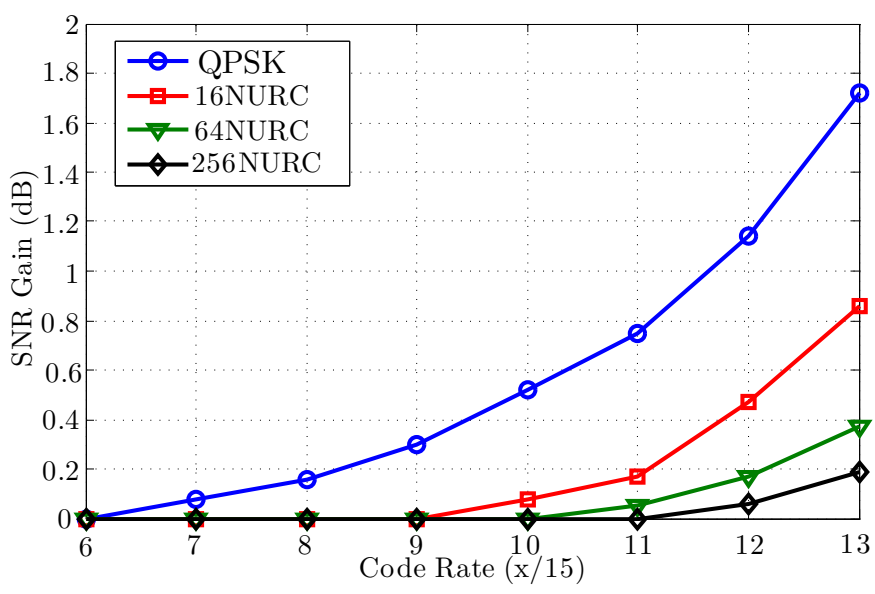

Fig. 8. Rotation SNR gain (dB) of 2D-NURCs, for i.i.d Rayleigh channel

Uniform QAM constellations use fixed rotation angles for each order of constellation. However, NUCs have different shape depending on the coding rate (or SNR) for which they are optimized. Hence, a different shape implies a different optimum rotation angle. Table IV shows the resulting rotation angles, for the orders of constellation (QPSK to 256QAM) and CRs (8/15 to 13/15) with potential SNR rotation gain. Those configurations with a SNR rotation gain under $0.1 \mathrm{~dB}$ are marked with a dash. In the Table IV it can be noted that the optimum rotation angle is higher for low-order constellations and high CRs. The same occurs for SNR gains, see Fig. 8.

The additional diversity introduced by RCs improves the performance of BICM for higher CRs, whereas for lower CRs it is preferable to rely on the error-correction capabilities of the FEC code [6]. With 2D-256NURCs, there is only gain with the two highest CRs (12/15 and 13/15). For CR 12/15, the optimum rotation angle obtained is $1^{\circ}$, but with a slight SNR gain of $0.03 \mathrm{~dB}$. For $13 / 15$, the SNR gain obtained is $0.2 \mathrm{~dB}$. The gain of RCs can be higher with more restrictive channels with erasures.

Regarding the demapping complexity, there is no increase when rotating 2D-NUCs. The QCSR demapper can be used in order to reduce the complexity in a similar way than in previous sections. The rotation does not influence the condensation, so this part of the algorithm remains identical. Only a slight change in the first stage of QSR is required, since it is necessary to reorganize the new $N$ rotated symbols by probability order, i.e., to obtain new histograms.

Fig. 9 shows the BER performance of the QCSR demapper with and without rotation, compared with the optimum ML demapper, for CRs $12 / 15$ and $13 / 15$. For CR 12/15, the SNR performance of QCSR is very similar with and without
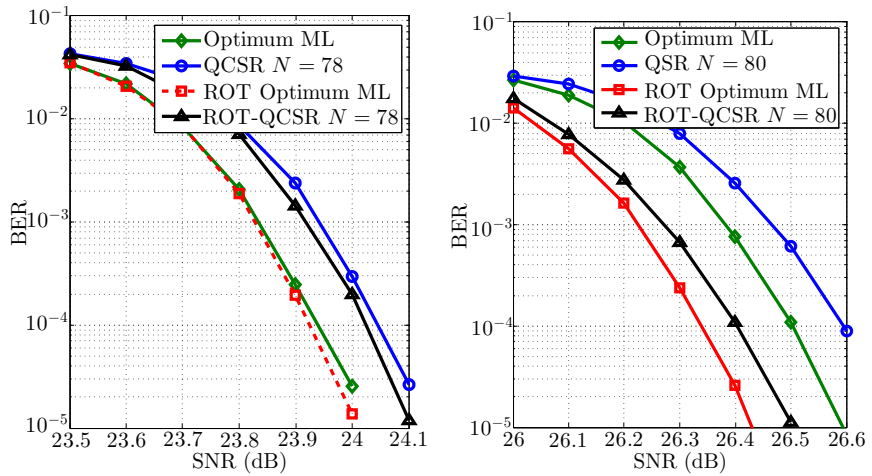

Fig. 9. Performance comparison of ML and QCSR demappers, with and without rotation. Results presented for 256QAM and code rates 12/15 (left) and 13/15 (right), i.i.d. Rayleigh channel.

TABLE $\mathrm{V}$

NUMBER OF DISTANCES AND COMPLEXITY REDUCTION OF QCSR WITH 2D-1KNUCS COMPARED TO THE ML DEMAPPER

\begin{tabular}{|c||c|c|c|c|c|}
\hline Code Rate & $2 / 15$ & $6 / 15$ & $8 / 15$ & $10 / 15$ & $13 / 15$ \\
\hline \hline$N_{Q S R}$ & 840 & 420 & 380 & 345 & 290 \\
\hline$N_{C S R}$ & 16 & 336 & 416 & 704 & 896 \\
\hline$N_{Q C S R}$ & 16 & 102 & 136 & 224 & 252 \\
\hline Reduction & $98 \%$ & $90 \%$ & $86 \%$ & $78 \%$ & $75 \%$ \\
\hline Gain (dB) compared to 1D & $<0$ & $<0$ & 0 & 0.12 & 0.15 \\
\hline
\end{tabular}

rotation. However, the same does not occur with 13/15, where the SNR gain of the QCSR rotated demapper is also $0.2 \mathrm{~dB}$ (as the rotated ML), and the number of distances is maintained, with $N=80$. Furthermore, compared to the non-rotated ML demapper, the QCSR rotated demapper has a $0.1 \mathrm{~dB}$ gain, and a complexity reduction of $69 \%$.

\section{QCSR with $2 D-1 k N U C$}

In this section, we extend the QCSR algorithm to 2D$1 \mathrm{kNUCs}$, for the CRs in which there is a potential SNR gain compared to $1 \mathrm{D}-1 \mathrm{kNUCs}$. Table $\mathrm{V}$ shows the number of distances calculated with the QCSR demapper for 2D1 kNUCs $\left(N_{Q C S R}\right)$ and the reduction percentage. The number of distances is obtained for different representative CRs: the lowest $2 / 15$, the highest $13 / 15$ and three medium CRs $6 / 15,8 / 15$ and $10 / 15$, and the proposed criteria is again to select the smallest possible $N$ that ensures a performance loss smaller than $0.1 \mathrm{~dB}$. With $2 \mathrm{D}-1 \mathrm{kNUCs}$, the number of required distances can be reduced from $75 \%$ to $98 \%$, with a higher reduction than $256 \mathrm{NUCs}$. In this case, the constellations remain condensed even for the highest CR. As occurred for 256NUCs, the maximum number of distances is obtained for the CR 13/15, with $N_{Q C S R}=252$. The number of Euclidean distances is always under 256, lower than 2D-256NUCs with optimum demapping.

Despite of the QCSR performance loss of $0.1 \mathrm{~dB}$, there is a SNR gain for several CRs compared to 1D-NUCs with optimum demapping. Table $\mathrm{V}$ shows the SNR gain of QCSR and $2 \mathrm{D}$ compared to the optimum 1D-demapper, where " $<0$ " means a SNR loss. Fig. 10 depicts an example of the BER performance obtained with CRs $6 / 15$ and $10 / 15$. With $10 / 15$, the SNR gain of QCSR can be easily observed. However, with 

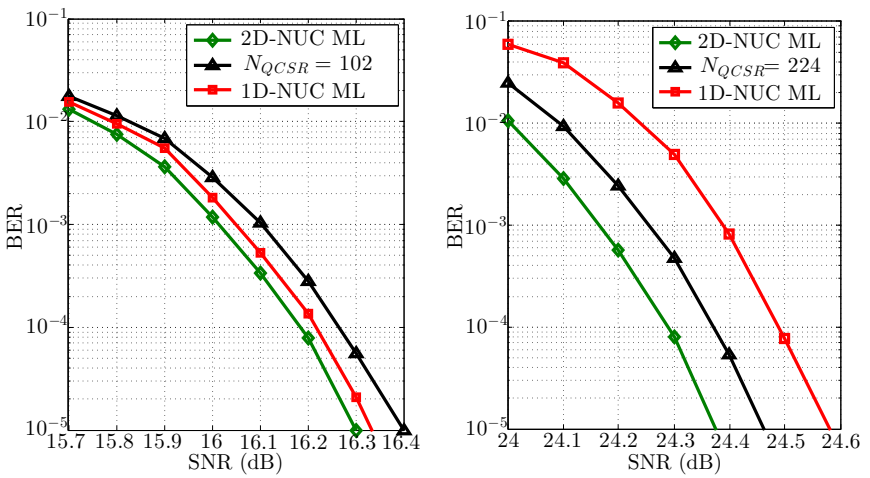

Fig. 10. Performance comparison of 1D- and 2D-NUC with the ML and QCSR demappers. Results presented for 1024QAM and code rates 6/15 (left) and 10/15 (right), i.i.d. Rayleigh channel.

6/15 it is better to keep 1D-NUCs instead, since the SNR required with the 1D-demapper is lower than the obtained with QCSR and 2D-NUCs, and also the complexity.

\section{CONCLUSION}

In this paper, a suboptimal demapper for 2D-NUCs has been proposed to reduce the demapping complexity in terms of distances required for the LLR computation. The proposed demapper is based on the combination of two strategies to take advantage of the symmetry of the constellations and the condensation, i.e., similar I/Q values of several constellation symbols, of NUCs at low SNRs. The results show that a reduction in the number of required operations from $69 \%$ to $93 \%$ can be achieved, depending on the code rate, when compared to the optimal Maximum Likelihood (ML) demapper, with almost no performance degradation (under $0.1 \mathrm{~dB}$ ).

\section{REFERENCES}

[1] A. Guillen i Fabregas, A. Martinez, and G. Caire, Bit-interleaved Coded Modulation, Foundations and Trends in Communications and Information Theory, vol. 5, no. 1-2, pp. 1-153, Nov. 2008.

[2] L. Michael and D. Gomez-Barquero, Bit-Interleaved Coding and Modulation (BICM) for ATSC 3.0, IEEE Trans. Broadcast., vol. 62, no. 1, March 2016

[3] U. Ladebusch and C. A. Liss, Terrestrial DVB (DVB-T): A Broadcast Technology for Stationary Portable and Mobile Use, Procedings of the IEEE, vol. 94, no. 1, pp. 183-193, Jan. 2006.

[4] T. Ikeda, Transmission System for ISDB-T $T_{S B}$ (Digital Terrestrial Sound Broadcasting), Procedings of the IEEE, vol. 94, no. 1, pp. 257-260, Jan. 2006.

[5] I. Eizmendi, et al., DVB-T2: The Second Generation of Terrestrial Digital Video Broadcasting System, IEEE Trans. Broadcast., vol. 60, no. 2, pp. 258-271, June 2014.

[6] D. Gozalvez, J. J. Gimenez, D. Gomez-Barquero, and N. Cardona, Rotated Constellations for Improved Time and Frequency Diversity in $D V B-N G H$, IEEE Trans. Broadcast., vol. 59, no. 2, pp. 298-305, June 2013.

[7] D. Gomez-Barquero, C. Douillard, P. Moss, and V. Mignone, DVB-NGH: The Next Generation of Digital Broadcast Services to Handheld Devices, IEEE Trans. Broadcast., vol. 60, no. 2, pp. 246-257, June 2014

[8] C. Douillard and C. A. Nour, Bit-Interleaved Coded Modulation in NextGeneration Mobile Broadcast Standard DVB-NGH, in Next Generation Mobile Broadcasting, D. Gómez-Barquero (ed.), CRC Press, 2013, pp. 321-354.

[9] P. Xue, K. Bae, K. Kim, and H. Yang, Best Candidates Based SoftDemapper for Rotated M-QAM Constellation, IEEE 11th Consumer Communications and Networking Conference (CCNC), Las Vegas, USA, Jan. 2014.
[10] L. Fay, L. Michael, D. Gomez-Barquero, N. Ammar, and W. Caldwell, An Overview of the ATSC 3.0 Physical Layer Specification, IEEE Trans. Broadcast., vol. 62, no. 1, March 2016.

[11] J. Stott, CM and BICM limits for rectangular constellations, BBC Research \& Development White Paper WHP 257, Aug. 2013.

[12] N. Loghin et al., Non-Uniform Constellations for ATSC 3.0, IEEE Trans. Broadcast., vol. 62, no. 1, March 2016.

[13] G. J. Foschini and S. B. Weinstein, Optimization of Two-Dimensional Signal-Constellations in the Presence of Gaussian Noise, IEEE Transactions on Communications, vol. com-22, no. 6, pp. 28-38, Jan. 1974.

[14] J. Zöllner and N. Loghin, Optimization of High-order Non-uniform QAM Constellations, IEEE International Symposium on Broadband Multimedia Systems and Broadcasting (BMSB), London, UK, June 2013.

[15] B. Mouhouche, D. Ansorregui, and A. Mourad, High Order NonUniform Constellations for broadcasting UHDTV, IEEE Wireless Communications and Networking Conference, Istambul, Turkey, April 2014.

[16] D. Perez-Calderon, V. Baena-Lecuyer, A. C. Oria, P. Lopez, and J. G. Doblado, Rotated constellation demapper for DVB-T2, IEEE Electronic Letters, vol. 47, pp. 31-32, Jan. 2011.

[17] D. Perez-Calderon, V. Baena-Lecuyer, A. C. Oria, P. Lopez, and J. G. Doblado, Simplified Rotated Constellation Demapper for Second Generation Terrestrial Digital Video Broadcasting, IEEE Trans. Broadcast., vol. 59, no. 1, pp. 160-167, March 2013.

[18] B. Hassibi and H. Vikalo, On the sphere-decoding algorithm I. Expected complexity, IEEE Tans. Signal Processing, vol. 53, no. 8, pp. 2806-2818, Aug. 2005.

[19] J. Stott, Beyond NUQAM \& ConQAM - overcoming their limitations, especially at lower SNRs, DVB document TM-T0007.

[20] J. Stott, BICM limits for Condensed QAM, Further results, DVB document TM-MIMO0023.

[21] S. Kwon, et al., Simplified Non-uniform Constellation Demapping Scheme for the Next Broadcasting System, IEEE International Symposium on Broadband Multimedia Systems and Broadcasting (BMSB), Ghent, Belgium, June 2015.

[22] ETSI TS 102831 V1.2.1, Digital Video Broadcasting (DVB); Implementation guidelines for a second generation digital terrestrial television broadcasting system (DVB-T2), Aug. 2012.

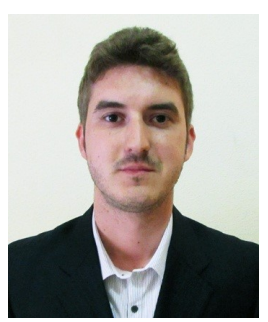

Manuel Fuentes received his M.Sc. degree in telecommunication engineering and a second M.Sc. degree in communication technologies, systems and networks from the Universitat Politecnica de Valencia, Spain, in 2012 and 2013, respectively. Currently, he is pursuing a $\mathrm{Ph} . \mathrm{D}$. degree in telecommunications at the Institute of Telecommunications and Multimedia Applications (iTEAM), where he is working since 2012. He participated in several R\&D projects where his research interests were focused on protection ratio measurements and network planning activities between digital broadcasting and 4G (LTE) technologies. He is a current member of the DVB European forum and the ATSC forum, contributing to the ATSC 3.0 standardization process. His current research interests include innovative techniques in bit-interleaved coding and modulation systems, such as non-uniform constellations or signal space diversity techniques, and multiantenna communications.

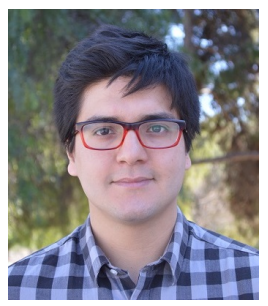

David Vargas received his M.Sc. degree in Telecommunication engineering from Universitat Politecnica de Valencia (UPV), Spain in 2009. During his studies he spent one year at the University of Turku (UTU), Finland. Currently he is pursuing a Ph.D. degree at the Mobile Communications Group at the Institute of Telecommunications and Multimedia Applications (iTEAM), UPV. He has been a guest researcher in the summer of 2011 at the Vienna University of Technology, Austria, during 2013 at McGill University, Montreal, Canada, and a research intern in 2015 at BBC Research \& Development, London, UK. He has participated in the standardization process of the next generation mobile broadcasting standard DVB-NGH and is currently an active participant in the standardization process of the next-generation terrestrial broadcasting standard ATSC 3.0. His research interests include multi-antenna communications, signal processing for communications, and digital broadcasting. 


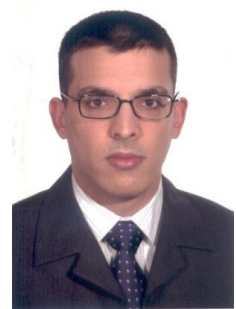

David Gómez-Barquero received the double M.Sc. degrees in telecommunications engineering from the Universitat Politecnica de Valencia (UPV), Spain, and the University of Gävle, Sweden, in 2004, and the Ph.D. degree in telecommunications from the UPV in 2009. He is a Senior Researcher (Ramon Cajal Fellow) with the Institute of Telecommunications and Multimedia Applications, UPV, where he leads a research group working on next generation broadcasting technologies. He is currently a Research Scholar with the New Jersey Institute of Technology, Newark, NJ, USA. Previously, he hold visiting research appointments at Ericsson Eurolab, Germany, the Royal Institute of Technology, Sweden, the University of Turku, Finland, the Technical University of Braunschweig, Germany, the Fraunhofer Heinrich Hertz Institute, Germany, and the Sergio Arboleda University of Bogota, Colombia. Since 2008, he has been actively participating in the European Digital Television Standardization Forum DVB in different topics such as upper layer forward error correction, DVB-T2, T2-Lite, and DVB-NGH. In 2013, he joined the U.S. Digital Television Standardization Forum ATSC to work on ATSC 3.0, where he is the ViceChairman of the Modulation and Coding Ad-Hoc Group. He is the Editor of the book entitled Next Generation Mobile Broadcasting (CRC Press). 